\title{
124.
}

\section{ON A PROPERTY OF THE CAUSTIC BY REFRACTION OF THE CIRCLE.}

[From the Philosophical Magazine, vol. vi. (1853), pp. 427-431.]

M. St LaURent has shown (Gergonne, vol. xviII. [1827] p. 1), that in certain cases the caustic by refraction of a circle is identical with the caustic of reflexion of a circle (the reflecting circle and radiant point being, of course, properly chosen), and a very elegant demonstration of M. St Laurent's theorems is given by M. Gergonne in the same volume, p. 48. A similar method may be employed to demonstrate the more general theorem, that the same caustic by refraction of a circle may be considered as arising from six different systems of a radiant point, circle, and index of refraction. The demonstration is obtained by means of the secondary caustic, which is (as is well known) an oval of Descartes. Such oval has three foci, any one of which may be taken for the radiant point: whichever be selected, there can always be found two corresponding circles and indices of refraction. The demonstration is as follows:-

Let $c$ be the radius of the refracting circle, $\mu$ the index of refraction; and taking the centre of the circle as origin, let $\xi, \eta$ be the coordinates of the radiant point, the secondary caustic is the envelope of the circle

$$
\mu^{2}\left(\overline{x-\alpha}^{2}+\overline{y-\beta}^{2}\right)-\left(\overline{\xi-\alpha}^{2}+\overline{\eta-\beta}^{2}\right)=0,
$$

where $\alpha, \beta$ are parameters which vary subject to the condition

$$
\alpha^{2}+\beta^{2}-c^{2}=0 ;
$$

the equation of the variable circle may be written

$$
\left\{\mu^{2}\left(x^{2}+y^{2}+c^{2}\right)-\left(\xi^{2}+\eta^{2}+c^{2}\right)\right\}-2\left(\mu^{2} x-\xi\right) \alpha-2\left(\mu^{2} y-\eta\right) \beta=0,
$$


which is of the form

$$
C+A \alpha+B \beta=0
$$

the envelope is therefore

$$
C^{2}=c^{2}\left(A^{2}+B^{2}\right) \text {. }
$$

Hence substituting, we have for the equation of the envelope, i.e. for the secondary caustic,

$$
\left\{\mu^{2}\left(x^{2}+\varkappa^{2}+c^{2}\right)-\left(\xi^{2}+\eta^{2}+c^{2}\right)\right\}^{2}=4 c^{2}\left\{\left(\mu^{2} x-\xi\right)^{2}+\left(\mu^{2} y-\eta\right)^{2}\right\},
$$

which may also be written

$$
\left\{\mu^{2}\left(x^{2}+y^{2}-c^{2}\right)-\left(\xi^{2}+\eta^{2}-c^{2}\right)\right\}^{2}=4 c^{2} \mu^{2}\left(\overline{x-\xi^{2}}+\overline{y-\eta}\right)^{2} ;
$$

and this may perhaps be considered as the standard form.

To show that this equation belongs to a Descartes' oval, suppose for greater convenience $\eta=0$, and write

$$
\mu^{2}\left(x^{2}+y^{2}-c^{2}\right)-\xi^{2}+c^{2}=2 c \mu \sqrt{(x-\xi)^{2}+y^{2}} ;
$$

multiplying this equation by $1-\frac{1}{\mu^{2}}$, and adding to each side $c^{2}\left(\mu-\frac{1}{\mu}\right)^{2}+(x-\xi)^{2}+y^{2}$, we have

$$
\begin{aligned}
& \left(1-\frac{1}{\mu^{2}}\right)\left\{\mu^{2}\left(x^{2}+y^{2}-c^{2}\right)-\xi^{2}+c^{2}\right\}+(x-\xi)^{2}+y^{2}+c^{2}\left(\mu-\frac{1}{\mu}\right)^{2} \\
& =(x-\xi)^{2}+y^{2}+2 c\left(\mu-\frac{1}{\mu}\right) \sqrt{(x-\xi)^{2}+y^{2}}+c^{2}\left(\mu-\frac{1}{\mu}\right)^{2}
\end{aligned}
$$

or reducing

$$
\mu^{2}\left\{\left(x-\frac{\xi}{\mu^{2}}\right)^{2}+y^{2}\right\}=\left\{\sqrt{(x-\xi)^{2}+y^{2}}+c\left(\mu-\frac{1}{\mu}\right)\right\}^{2} ;
$$

again, multiplying the same equation by $\frac{1}{\mu^{2}}\left(1-\frac{c^{2}}{\xi^{2}}\right)$, and adding to each side

$$
\frac{\xi^{2}}{\mu^{2}}\left(1-\frac{c^{2}}{\xi^{2}}\right)^{2}+\frac{c^{2}}{\xi^{2}}\left(\overline{x-\xi^{2}}+y^{2}\right)
$$

we have

$$
\begin{aligned}
& \frac{1}{\mu^{2}}\left(1-\frac{c^{2}}{\xi^{2}}\right)\left\{\mu^{2}\left(x^{2}+y^{2}-c^{2}\right)-\xi^{2}+c^{2}\right\}+\frac{c^{2}}{\xi^{2}}\left(x-\xi^{2}+y^{2}\right)+\frac{\xi^{2}}{\mu^{2}}\left(1-\frac{c^{2}}{\xi^{2}}\right)^{2} \\
& =\frac{c^{2}}{\xi^{2}}\left((x-\xi)^{2}+y^{2}\right)+\frac{2 c}{\mu}\left(1-\frac{c^{2}}{\xi^{2}}\right) \sqrt{(x-\xi)^{2}+y^{2}}+\frac{\xi^{2}}{\mu^{2}}\left(1-\frac{c^{2}}{\xi^{2}}\right)^{2} ;
\end{aligned}
$$

or reducing,

$$
\left(x-\frac{c^{2}}{\xi}\right)^{2}+y^{2}=\left\{\frac{c}{\xi} \sqrt{(x-\xi)^{2}+y^{2}}+\frac{\xi}{\mu}\left(1-\frac{c^{2}}{\xi^{2}}\right)\right\}^{2} .
$$


Hence, extracting the square roots of each side of the equations thus found, we have the equation of the secondary caustic in either of the forms

$$
\begin{aligned}
& \sqrt{\left(x-\frac{\xi}{\mu^{2}}\right)^{2}+y^{2}}=\frac{1}{\mu} \sqrt{(x-\xi)^{2}+y^{2}}+\frac{c}{\mu}\left(\mu-\frac{1}{\mu}\right) \\
& \sqrt{\left(x-\frac{c^{2}}{\xi}\right)^{2}+y^{2}}=\frac{c}{\xi} \sqrt{(x-\xi)^{2}+y^{2}}+\frac{1}{\mu}\left(\xi-\frac{c^{2}}{\xi}\right)
\end{aligned}
$$

to which are to be joined

$$
\begin{gathered}
\sqrt{\left(x-\frac{c^{2}}{\xi}\right)^{2}+y^{2}}=\frac{c \mu}{\xi} \sqrt{\left(x-\frac{\xi}{\mu^{2}}\right)^{2}+y^{2}}+\frac{\xi}{\mu}-c^{c^{2} \mu} \\
c\left(\mu-\frac{1}{\mu}\right) \sqrt{\left(x-\frac{c^{2}}{\xi}\right)^{2}+y^{2}}+\left(-\xi+\frac{c^{2}}{\xi}\right) \sqrt{\left(x-\frac{\xi}{\mu^{2}}\right)^{2}+y^{2}}+\left(\frac{\xi}{\mu}-\frac{c^{2} \mu}{\xi}\right) \sqrt{(x-\xi)^{2}+y^{2}}=0 .
\end{gathered}
$$

Write successively,

$$
\begin{array}{lll}
\xi^{\prime}=\xi, & c^{\prime}=c, & \mu^{\prime}=\mu, \\
\xi^{\prime}=\frac{c^{2}}{\xi}, & c^{\prime}=\frac{c}{\mu}, & \mu^{\prime}=\frac{c}{\xi}, \\
\xi^{\prime}=\frac{\xi}{\mu^{2}}, & c^{\prime}=\frac{c}{\mu}, & \mu^{\prime}=\frac{1}{\mu}, \\
\xi^{\prime}=\xi, & c^{\prime}=\frac{\xi}{\mu}, & \mu^{\prime}=\frac{\xi}{c}, \\
\xi^{\prime}=\frac{c^{2}}{\xi}, & c^{\prime}=c, & \mu^{\prime}=\frac{c \mu}{\xi}, \\
\xi^{\prime}=\frac{\xi}{\mu^{2}}, & c^{\prime}=\frac{\xi}{\mu}, & \mu^{\prime}=\frac{\xi}{c \mu} ;
\end{array}
$$

or, what is the same thing,

$$
\begin{array}{lll}
\xi=\xi^{\prime}, & c=c^{\prime}, & \mu=\mu^{\prime}, \\
\xi=\frac{\xi^{\prime}}{\mu^{\prime 2}}, & c=\frac{\xi^{\prime}}{\mu^{\prime}}, & \mu=\frac{\xi^{\prime}}{c^{\prime} \mu^{\prime}}, \\
\xi=\frac{\xi^{\prime}}{\mu^{\prime 2}}, & c=\frac{c^{\prime}}{\mu^{\prime}}, & \mu=\frac{1}{\mu^{\prime}}, \\
\xi=\xi^{\prime}, & c=\frac{\xi^{\prime}}{\mu^{\prime}}, & \mu=\frac{\xi^{\prime}}{c^{\prime}}, \\
\xi=\frac{c^{\prime 2}}{\xi^{\prime}}, & c=c^{\prime}, & \mu=\frac{c^{\prime} \mu^{\prime}}{\xi^{\prime}}, \\
\xi=\frac{c^{\prime 2}}{\xi^{\prime}}, & c=\frac{c^{\prime}}{\mu^{\prime}}, & \mu=\frac{c^{\prime}}{\xi^{\prime}} ;
\end{array}
$$



or, again,

$$
\begin{array}{lll}
\xi^{\prime}=\xi, & \frac{c^{\prime 2}}{\xi^{\prime}}=\frac{c^{2}}{\xi}, & \frac{\xi^{\prime}}{\mu^{\prime 2}}=\frac{\xi}{\mu^{2}}, \\
\xi^{\prime}=\frac{c^{2}}{\xi}, & \frac{c^{\prime 2}}{\xi^{\prime}}=\xi, & \frac{\xi^{\prime}}{\mu^{\prime 2}}=\xi, \\
\xi^{\prime}=\frac{\xi}{\mu^{2}}, & \frac{c^{\prime 2}}{\xi^{\prime}}=\frac{c^{2}}{\xi}, & \frac{\xi^{\prime}}{\mu^{\prime 2}}=\xi, \\
\xi^{\prime}=\xi, & \frac{c^{\prime 2}}{\xi^{\prime}}=\frac{\xi}{\mu^{2}}, & \frac{\xi^{\prime}}{\mu^{\prime 2}}=\frac{c^{2}}{\xi}, \\
\xi^{\prime}=\frac{c^{2}}{\xi}, & \frac{c^{\prime 2}}{\xi^{\prime}}=\xi, & \frac{\xi^{\prime}}{\mu^{2}}=\frac{\xi}{\mu^{2}}, \\
\xi^{\prime}=\frac{\xi}{\mu^{2}}, & \frac{c^{\prime 2}}{\xi^{\prime}}=\xi, & \frac{\xi^{\prime}}{\mu^{\prime 2}}=\frac{c^{2}}{\xi} ;
\end{array}
$$

then, whichever system of values of $\xi^{\prime}, c^{\prime}, \mu^{\prime}$ be substituted for $\xi, c, \mu$, we have in each case identically the same secondary caustic, the effect of the substitution being simply to interchange the different forms of the equation; and we have therefore identically the same caustic. By writing

$$
\begin{array}{r}
\left(\xi^{\prime}, c^{\prime}, \mu^{\prime}\right)=(\xi, c, \mu) \\
=\alpha(\xi, c, \mu), \\
\& c .,
\end{array}
$$

$\alpha, \beta, \gamma, \delta, \epsilon$ will be functional symbols, such as are treated of in my paper "On the Theory of Groups as depending on the symbolic equation $\theta^{n}=1$," [125], and it is easy to verify the equations

$$
\begin{aligned}
& 1=\alpha \beta=\beta \alpha=\gamma^{2}=\delta^{2}=\epsilon^{2}, \\
& \alpha=\beta^{2}=\delta \gamma=\epsilon \delta=\gamma \epsilon, \\
& \beta=\alpha^{2}=\epsilon \gamma=\gamma \delta=\delta \epsilon, \\
& \gamma=\delta \alpha=\epsilon \beta=\beta \delta=\alpha \epsilon, \\
& \delta=\epsilon \alpha=\gamma \beta=\alpha \gamma=\beta \epsilon, \\
& \epsilon=\gamma \alpha=\delta \beta=\beta \gamma=\alpha \delta .
\end{aligned}
$$

Suppose, for example, $\xi=-c$, i.e. let the radiant point be in the circumference; then in the fourth system $\xi^{\prime}=-c, c^{\prime}=-\frac{c}{\mu}$, (or, since $c^{\prime}$ is the radius of a circle, this radius may be taken $\frac{c}{\mu}$ ), $\mu^{\prime}=-1$, or the new system is a reflecting system. This is one of M. St Laurent's theorems, viz.

C. II. 
Theorem. The caustic by refraction of a circle when the radiant point is on the circumference, is the caustic by reflexion for the same radiant point, and a concentric circle the radius of which is the radius of the first circle divided by the index of refraction.

Again, if $\xi=-c \mu$, the fifth system gives $\xi^{\prime}=\frac{c^{2}}{\xi^{\prime}}, c^{\prime}=c, \mu^{\prime}=-1$, or the new system is in this case also a reflecting system. This is the other of M. St Laurent's theorems, viz. :-

THEOREM. The caustic by refraction of a circle when the distance of the radiant point from the centre is equal to the radius of the circle multiplied by the index of refraction, is the caustic by reflexion of the same circle for a radiant point which is the image of the first radiant point.

Of course it is to be understood that the image of a point means a point whose distance from the centre $=$ square of radius $\div$ distance.

2 Stone Buildings, Nov. 2, 1853. 